\title{
The warm absorber and X-ray variability of the Seyfert 1 galaxy NGC 3516 as seen by the XMM-Newton RGS
}

\author{
M. Mehdipour, G. Branduardi-Raymont, and M. J. Page
}

Mullard Space Science Laboratory, University College London, Holmbury St. Mary, Dorking, Surrey, RH5 6NT, UK

e-mail: missagh.mehdipour@ucl.ac.uk

Received 1 August 2009 / Accepted 17 February 2010

\section{ABSTRACT}

\begin{abstract}
Aims. We present a new analysis of the soft and medium energy X-ray spectrum of the Seyfert 1 galaxy NGC 3516 taken with the Reflection Grating Spectrometer (RGS) and European Photon Imaging Camera (EPIC) on board the XMM-Newton observatory. We examine four observations made in October 2006. We investigate whether the observed variability is due to absorption by the warm absorber and/or is intrinsic to the source emission.

Methods. We analyse in detail the EPIC-pn and RGS spectra of each observation separately.

Results. The warm absorber in NGC 3516 is found to consist of three phases of ionisation, two of which have outflow velocities of more than $1000 \mathrm{~km} \mathrm{~s}^{-1}$. The third phase (the least ionised one) is much slower at $100 \mathrm{~km} \mathrm{~s}^{-1}$. One of the high ionisation phases, with $\log \xi$ of 2.4, is found to have a partial covering fraction of about $60 \%$. It has previously been suggested that the passage of a cloud, part of a disc wind, in front of the source (producing a change in the covering fraction) was the cause of a significant dip in the lightcurve during one of the observations. From our modelling of the EPIC-pn and RGS spectra, we find that variation in the covering fraction cannot be solely responsible for this. We show that intrinsic change in the source continuum plays a much more significant role in explaining the observed flux and spectral variability than originally thought.
\end{abstract}

Key words. galaxies: active - galaxies: Seyfert - galaxies: individual: NGC 3516 - X-rays: galaxies - techniques: spectroscopic

\section{Introduction}

Recent X-ray observations of many Seyfert 1 galaxies and some quasars show evidence of absorbing photoionised gas in our line of sight towards the active nucleus (e.g. Kaastra et al. 2000; Reeves et al. 2003; Blustin et al. 2005; Costantini et al. 2007; Kaastra 2008). This gas is normally found to be outflowing with often several distinct components, and is referred to as a "warm absorber"; its absorption signatures are clearly seen in the high resolution X-ray spectra. Active galactic nucleus (AGN) outflows, in general, are believed to have important astrophysical implications. For example, strong outflows from the AGN can affect the growth of the supermassive black hole at its core and enrichment of the interstellar medium and the intergalactic medium could alter the course of the host galaxy evolution through feedback processes. The extent of the outflow contribution depends on its kinetic luminosity and the mass outflow rate. To reconstruct the kinetic luminosity of the outflow, the ionisation state and structure of the warm absorber need be to determined from photoionisation modelling of the high resolution $\mathrm{X}$-ray spectra.

The first warm absorber identification was reported by Halpern (1984) from an observation of the QSO MR2251-178 with the Einstein Observatory. Then in the days of ROSAT and $A S C A$, warm absorbers were found through the detection of what was interpreted as broad and blended continuum absorption edges of ions such as O VII and O VIII. For examples of early studies of AGN warm absorbers using ROSAT see Nandra \& Pounds (1992), Nandra et al. (1993) and Turner et al. (1993); for those using ASCA, see Reynolds (1997) and George et al. (1998). However, the instruments used at that time had insufficient spectral resolution to measure any details, like the presence and parameters of narrow absorption lines. With the advent of high resolution X-ray spectrometers onboard XMM-Newton and Chandra, the availability of high-resolution spectra has allowed a major leap forward in the study of AGN outflows in the last decade, starting with the original discovery of numerous blue-shifted absorption lines from photoionised, outflowing gas in NGC 5548 by Kaastra et al. (2000), the subsequent identification of similar features in a number of AGN and the discovery of an unresolved transition array (UTA) of low ionised iron ions in IRAS $13349+2438$ by Sako et al. (2001).

The source NGC 3516 is a Seyfert 1.5 SB0 galaxy at a redshift of 0.008836 (Keel 1996) in the constellation of Ursa Major. Even from early X-ray observations of this object, signatures of a multi-phase warm absorber have been evident. From simultaneous far-UV and ASCA X-ray observations in 1995, a warm absorber with at least two absorption components was reported by Kriss et al. (1996). The two components differ by a factor of 8 in the ionisation parameter; the more highly ionised has a column density twice as large as the less ionised component. Netzer et al. (2002) investigated spectral variations of NGC 3516 over a period of seven years, by using archival ASCA and early Chandra observations. They reported a large drop in flux (factor of $\sim 50$ at $1 \mathrm{keV}$ ) between an ASCA observation in 1994 and the Chandra observation in 2000. Netzer et al. (2002) concluded that the variations in the observed flux and spectra at these epochs were consistent with a constant column density of line-of-sight material reacting to changes in the ionising continuum.

The galaxy NGC 3516 was observed twice by XMM-Newton in April and November 2001; both observations were partially overlapping with Chandra observations. Turner et al. (2002) presented results from the simultaneous Chandra High Energy Transmission Grating (HETG) and XMM-Newton observations made in November 2001: analysis of the $\mathrm{Fe} \mathrm{K} \alpha$ regime showed 
evidence of several narrow emission features and of rapid evolution of the $\mathrm{Fe} \mathrm{K} \alpha$ line during the observation. From the $2001 \mathrm{ob}-$ servations, Turner et al. (2005) reported three distinct zones (phases) of gas with different ionisation parameters covering the active nucleus. The ionisation parameter $(\xi)$ is defined as

$\xi=\frac{L_{\text {ion }}}{n r^{2}}$,

where $L_{\text {ion }}$ is the luminosity of the ionising source over the $1-1000$ Ryd band (in erg s${ }^{-1}$ ), $n$ the hydrogen number density (in $\mathrm{cm}^{-3}$ ) and $r$ the distance between the ionised gas and the ionising source (in cm). Therefore, $\xi$ is in units of erg $\mathrm{cm} \mathrm{s}^{-1}$.

The warm absorber phases found in Turner et al. (2005) are a low-ionisation UV/X-ray absorber with $\log \xi \sim-0.5$ and hydrogen column density of $N_{\mathrm{H}} \sim 0.5 \times 10^{22} \mathrm{~cm}^{-2}$; a more highly ionised gas with $\log \xi \sim 3.0$ and $N_{\mathrm{H}} \sim 2 \times 10^{22} \mathrm{~cm}^{-2}$ outflowing at a velocity of $\sim 1100 \mathrm{~km} \mathrm{~s}^{-1}$; and a phase with $\log \xi \sim 2.5$ and a very large hydrogen column density of $N_{\mathrm{H}} \sim 25 \times 10^{22} \mathrm{~cm}^{-2}$ covering $\sim 50 \%$ of the continuum. Turner et al. (2005) found the spectral variability in the 2001 observations to be consistent with the ionisation-state of the absorbing gas layers responding to the continuum flux variation.

More recently, four observations of NGC 3516 were performed by XMM-Newton in October 2006, interwoven with Chandra observations. The analysis of the EPIC-pn and Chandra HETG data is published in Turner et al. (2008). They discovered a previously unknown ionisation phase in the Fe K $\alpha$ regime, with $\log \xi \sim 4.3$ and $N_{\mathrm{H}} \sim 26 \times 10^{22} \mathrm{~cm}^{-2}$. In line with the results from the 2001 observations, a phase with a covering fraction of $\sim 50 \%$ was confirmed. This phase was found to have $\log \xi \sim 2.2$ and $N_{\mathrm{H}} \sim 20 \times 10^{22} \mathrm{~cm}^{-2}$. The source showed significant flux variability between observations, especially a dip in the lightcurve of the third observation. Turner et al. (2008) concluded that changes in the covering fraction of the phase partially covering the continuum provide a simple explanation of the dip in the lightcurve. They interpreted this as an eclipse of the continuum due to passage of a cloud across the line of sight over half a day.

We present a new analysis of the EPIC-pn, RGS and OM data from the four XMM-Newton observations of October 2006, with the emphasis put on the RGS spectra. Section 2 describes the observations and data analysis and Sect. 3 focuses on the lightcurves; the spectral modelling is described in detail in Sects. 4 and 5; we discuss our findings in Sect. 6 and give concluding remarks in Sect. 7.

\section{Observations and data analysis}

We extracted the EPIC-pn, RGS and OM data for the four 2006 observations from the XMM-Newton Science Archive (XSA). The XMM-Newton observation IDs are 04012010401 (51.6 ks), 04012010501 (68.8 ks), 04012010601 (68.2 ks) and $04012011001(67.8 \mathrm{ks})$. Hereafter, we refer to them as Obs. 1, Obs. 2, Obs. 3 and Obs. 4, respectively.

All the Observation Data File (ODF) data were processed using SAS v7.12, unless specified otherwise in the text. For EPIC-pn, RGS1 and RGS2, periods of high flaring background were filtered out before extracting scientific products. For the EPIC-pn, this was done by creating Good Time Intervals (GTIs), and using them in conjunction with the internal GTI tables to remove periods with single event count rates exceeding 0.4 counts per second in the energy range $E>10 \mathrm{keV}$. For the RGS instrument, in addition to the internal GTIs, we constructed an extra GTI table to filter out periods with count rates above 0.4 counts per second in CCD number 9 , which is the closest to the optical axis of the telescope and therefore the most affected by background flares.

The spectra and lightcurves of EPIC-pn, operating in the Small Window mode, were extracted from a circular region of $40^{\prime \prime}$ radius centred on the source. The background was extracted from a source-free region of the same radius, located at the same RAWY as the source on the same chip. The EPIC-pn showed no evidence of pile-up. The EPIC-pn lightcurves were backgroundsubtracted and corrected (using the epiclccorr SAS v8 task) for various effects on the detection efficiency such as vignetting, bad pixels, chip gaps, Point Spread Function (PSF) variation and quantum efficiency; the task also makes corrections, which vary with time and thus affect the stability of the detection, like dead time and GTIs. The RGS instruments were operated in the standard Spectro+Q mode. The first and second order spectra of RGS1 and RGS2 were extracted, and the response matrices were generated with the rgsproc processing task.

The OM was operated in Image+Fast mode in all observations, as well as in Science User Defined mode in the last two exposures of Obs. 1, Obs. 3 and Obs. 4. The images in all four observations were taken with the $U$ filter (3000-3900 ̊). The OM Image mode data were processed with the omichain pipeline. The resulting images are each of $1400 \mathrm{~s}$ exposure. The FWHM of the source is about $1.6^{\prime \prime}$, which is consistent with the OM on-board PSF FWHM of $1.55^{\prime \prime}$ in the $U$ filter. We performed photometry on each image in a fully interactive way using the omsource program: we carefully selected the source and background regions to extract the count rate, and applied all the necessary corrections such as for the PSF and coincidence losses, including time-dependent sensitivity (TDS) corrections. The OM lightcurves were extracted from a circle of 8 pixels radius ( 1 pixel $=0.48^{\prime \prime}$ ) centred on the source nucleus. The background was extracted from a source-free region of the same radius.

All the results presented in this work are from simultaneous fits of the EPIC-pn and the first-order RGS1 and RGS2 spectra, unless specified otherwise in the text. The EPIC-pn spectra are in the range $0.2-10 \mathrm{keV}$ and are binned to a minimum of 200 counts per bin. The RGS data are in the range 6-38 $\AA$ (0.33-2.07 keV), binned by a factor of 3 (this corresponds to an average bin size of $0.04 \AA$, which still over-samples the RGS resolution element of $\sim 0.07 \AA F W H M$ ). All the spectra shown in this paper are background-subtracted and are in the observed frame. The spectral analysis and modelling was done using the SPEX (version 2.01.02) fitting code (Kaastra et al. 1996). All the parameter errors quoted in this paper correspond to a $\Delta \chi^{2}$ of 2 .

\section{X-ray and UV lightcurves}

Figure 1 shows the OM (UV, top) and EPIC-pn (X-ray) lightcurves of the four observations. The source shows significant X-ray flux variability between observations, notably the flare in Obs. 1 and the dip in Obs. 3. From visual inspection of the X-ray lightcurves, the variations in the $0.2-2.0 \mathrm{keV}$ (soft X-ray) and 2.0-10.0 keV (hard X-ray) bands follow each other closely; however in Obs. 3 the ratio of soft to hard X-ray count rates clearly increases as the observation progresses, which indicates spectral variability; some evidence for a softer spectrum when brighter is also present in Obs. 1 and Obs. 4.

In contrast to the X-ray, the UV lightcurve shows no significant flaring in Obs. 1 and there is no a significant dip in the 


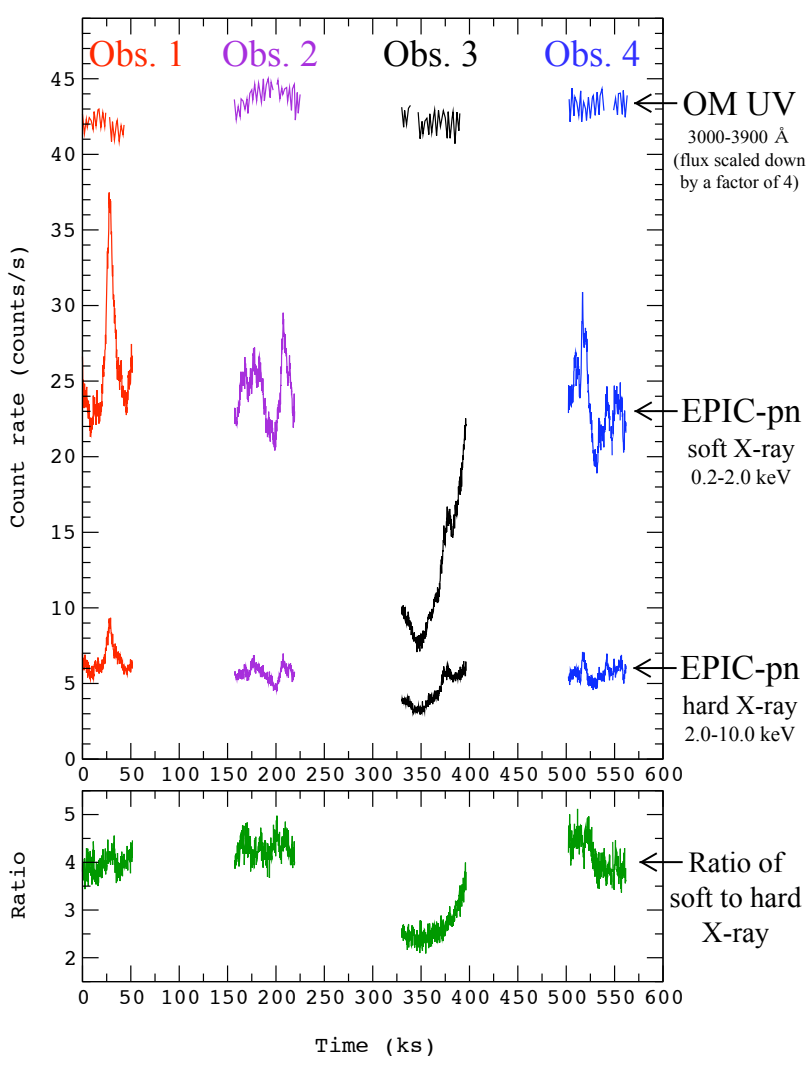

Fig. 1. Upper panel: top lightcurves: the OM background-subtracted $U$ band (3000-3900 ̊) lightcurves of Obs. 1 (red), Obs. 2 (purple), Obs. 3 (black) and Obs. 4 (blue); each count rate is scaled down by a factor of 4 for clarity of display and corresponds to an OM exposure of $1400 \mathrm{~s}$. Middle lightcurves: the EPIC-pn background-subtracted lightcurves of the four observations in $200 \mathrm{~s}$ time bins over $0.2-2.0 \mathrm{keV}$. Bottom lightcurves: the EPIC-pn background-subtracted lightcurves of the four observations in $200 \mathrm{~s}$ time bins over $2.0-10.0 \mathrm{keV}$. Lower panel (green): the ratio of $0.2-2.0 \mathrm{keV}$ (soft X-ray) to $2.0-10.0 \mathrm{keV}$ (hard $\mathrm{X}$-ray) lightcurves.

UV flux in Obs. 3; it is not unusual to see variability in the X-rays, and none in the UV (e.g. see Blustin et al. 2002, for analysis of NGC 3783).

\section{Preliminary modelling of the X-ray continuum and $\mathrm{Fe} \mathrm{K} \alpha$ line}

Figure 2 depicts an overview of the EPIC-pn and RGS spectra of the four observations: while Obs. 2 and 4 display very similar spectra (so much so that they are practically indistinguishable), that of Obs. 1 lies slightly above them, and that of Obs. 3 is clearly much fainter than all the others. The features of the spectra, in particular during the dip in Obs. 3, are discussed in sections below.

We started modelling the 4.0-10.0 keV EPIC-pn spectrum of Obs. 2 (which is the longest of the four observations) by a simple power-law (pow) redshifted to $z=0.008836$. The transmission of the Galactic neutral absorption was included by applying the hot model (collisional ionisation equilibrium) in SPEX. The Galactic H I column density in our line of sight was fixed to $N_{\mathrm{H}}=3.45 \times 10^{20} \mathrm{~cm}^{-2}$ (Kalberla et al. 2005) and the gas temperature to $0.5 \mathrm{eV}$ to mimic a neutral gas (Kaastra et al. 2009). The prominent $\mathrm{Fe} \mathrm{K} \alpha$ emission line at $\sim 6.4 \mathrm{keV}$ was modelled by a simple Gaussian profile (Gaus) with its width left free to vary. The parameters obtained from the preliminary modelling

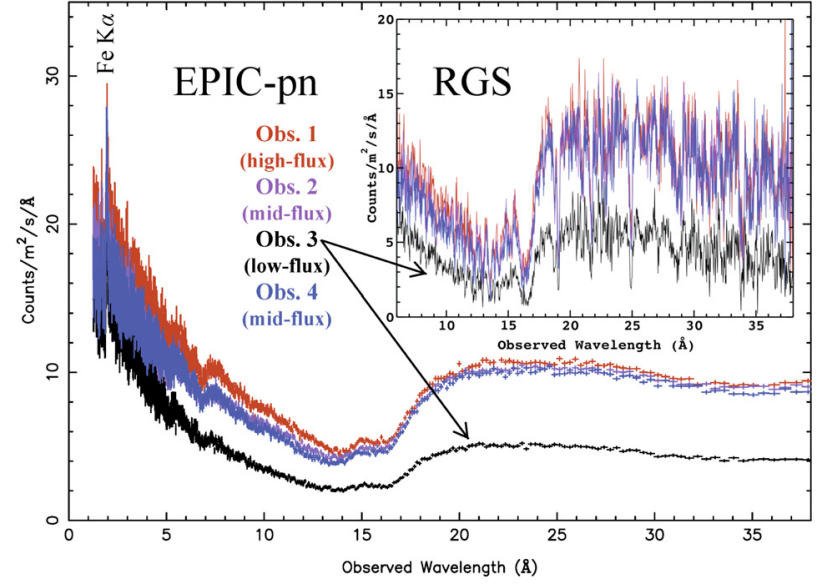

Fig. 2. EPIC-pn spectra of Obs. 1 (red), Obs. 2 (purple), Obs. 3 (black) and Obs. 4 (blue) binned to a minimum of 200 counts per bin. In the inset are the fluxed, combined RGS1 and RGS2 spectra binned by a factor of 3. Note that Obs. 2 and Obs. 4 are practically indistinguishable.

of the continuum and $\mathrm{Fe} \mathrm{K} \alpha$ line are shown in Table 1. Interestingly, we find that whereas the power-law flux falls significantly in Obs. 3, that of the $\mathrm{Fe} \mathrm{K} \alpha$ emission line remains unchanged compared to the other observations.

Figure 3 shows the 4.0-10.0 keV EPIC-pn fit, extrapolated to lower energies, displaying the presence of a soft excess and additional absorption in the $0.6-2.5 \mathrm{keV}$ band. The latter is most likely due to the warm absorber and will be modelled in the next section, by fitting the RGS and EPIC-pn data simultaneously.

To include modelling of the soft excess, we refitted the EPIC-pn spectrum of Obs. 2 over the $0.2-10.0 \mathrm{keV}$ energy range. We found that the power-law continuum model cannot fit the spectrum at all; a reduced Chi-squared $\left(\chi_{v}^{2}\right)$ value of 143 is obtained for 1111 degrees of freedom (d.o.f.). We then added a modified black body component $(m b b)$ to the continuum in addition to the power-law. The $m b b$ model describes the spectrum of a black body modified by coherent Compton scattering, and is often used to represent the accretion disk spectra of AGN (Kaastra \& Barr 1989). The addition of the $m b b$ component $(k T=128 \mathrm{eV})$ caused the $\chi_{v}^{2}$ to fall from 143 (1111 d.o.f.) to 21.0 (1109 d.o.f.). This is not yet a satisfactory fit, which shows the need for further modelling of the absorption in the $0.6-2.5 \mathrm{keV}$ range (i.e. the warm absorber); this is discussed in the following section.

\section{Spectral modelling of the warm absorber}

In the following subsections we describe the process of fitting the spectra with a model including warm absorption. We start by fitting in detail the spectrum of Obs. 2, which represents an "average" state of the source. In Sect. 5.1 we fit only the RGS spectrum of Obs. 2 and in Sect. 5.2 both the RGS and EPIC-pn simultaneously; we then proceed to fit the spectra of all the other observations.

\subsection{Spectral fit using a thin slab absorption model}

We began the analysis of the warm absorber by identifying and characterising the main absorption lines in the RGS spectrum of Obs. 2, shown in Fig. 4. The slab model in SPEX calculates the transmission through a slab of material with adjustable ionic column densities. We started by adding a slab component to the 
Table 1. Power-law and Fe K $\alpha$ emission line parameters, obtained from preliminary EPIC-pn fits (including the Galactic absorption) of all four observations over the $4.0-10 \mathrm{keV}$ energy range.

\begin{tabular}{|c|c|c|c|c|c|c|c|c|c|}
\hline \multirow[b]{2}{*}{ Obs. } & \multicolumn{2}{|c|}{ Power-law: } & \multirow[b]{2}{*}{$E_{0}{ }^{b}$} & \multirow[b]{2}{*}{$E^{c}$} & \multirow{2}{*}{\multicolumn{2}{|c|}{$\begin{array}{l}\mathrm{Fe} \mathrm{K} \alpha: \\
F W H M^{e}\end{array}$}} & \multirow[b]{2}{*}{$\sigma_{v}^{f}$} & \multirow[b]{2}{*}{ Normalisation $^{g}$} & \multirow[b]{2}{*}{$\chi_{v}^{2} /$ d.o.f. } \\
\hline & Photon Index $(\Gamma)$ & Normalisation $^{a}$ & & & & & & & \\
\hline 1 & $1.85 \pm 0.02$ & $2.9 \pm 0.1$ & 6.39 & $6.37 \pm 0.02$ & $+900_{-900}^{+1000}$ & $280 \pm 70$ & $6000 \pm 2000$ & $1.1 \pm 0.1$ & $1.54 / 330$ \\
\hline 2 & $1.82 \pm 0.02$ & $2.5 \pm 0.1$ & 6.39 & $6.38 \pm 0.01$ & $+500_{-500}^{+400}$ & $290 \pm 40$ & $6000 \pm 1000$ & $1.3 \pm 0.1$ & $1.42 / 361$ \\
\hline 3 & $1.75 \pm 0.02$ & $1.9 \pm 0.1$ & 6.39 & $6.38 \pm 0.01$ & $+500_{-500}^{+400}$ & $290 \pm 40$ & $6000 \pm 1000$ & $1.3 \pm 0.1$ & $1.58 / 324$ \\
\hline 4 & $1.84 \pm 0.02$ & $2.6 \pm 0.1$ & 6.39 & $6.40 \pm 0.01$ & $-500_{-400}^{+500}$ & $260 \pm 40$ & $5000 \pm 1000$ & $1.2 \pm 0.1$ & $1.38 / 311$ \\
\hline
\end{tabular}

Notes. ${ }^{(a)} 10^{51}$ photons s${ }^{-1} \mathrm{keV}^{-1}$ at $1 \mathrm{keV}$; ${ }^{(b)}$ theoretical rest frame wavelength in $\mathrm{keV}$; ${ }^{(c)}$ wavelength in the rest frame of NGC 3516 in $\mathrm{keV}$; ${ }^{(d)} \mathrm{km} \mathrm{s}^{-1} ;{ }^{(e)} \mathrm{eV} ;{ }^{f}$ rms velocity in $\mathrm{km} \mathrm{s}^{-1}\left(\sigma_{v}=F W H M / \sqrt{\ln 256}\right) ;{ }^{(g)} 10^{49}$ photons s${ }^{-1}$.

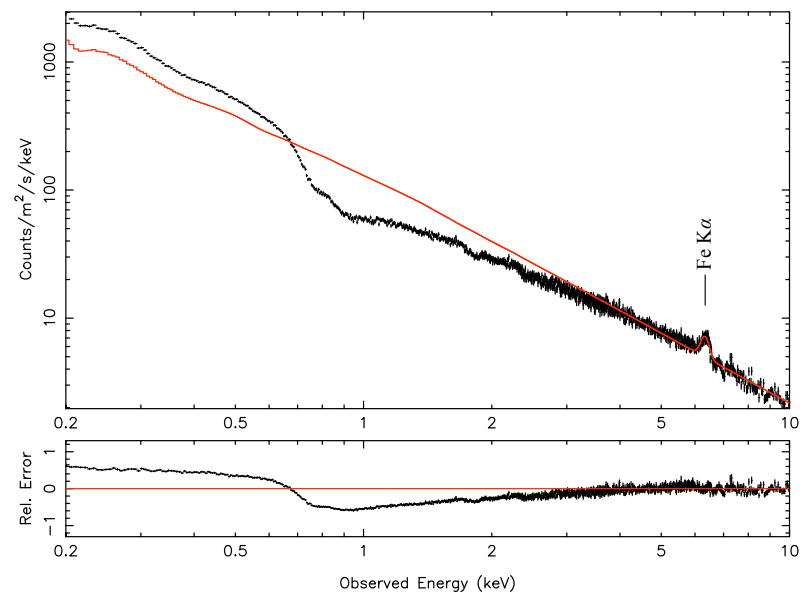

Fig. 3. Observation 2 EPIC-pn power-law fit (including the Gaussian Fe K $\alpha$ line and Galactic absorption) over the 4.0-10.0 keV range. The fit is extrapolated to lower energies, displaying the presence of a soft excess and additional absorption in the $0.6-2.5 \mathrm{keV}$ range.
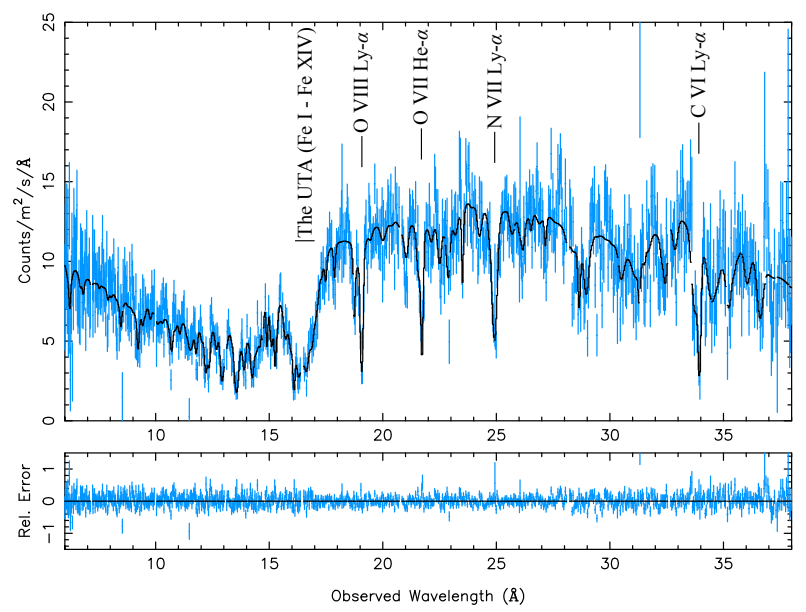

Fig. 4. RGS spectrum of Obs. 2, fitted using the slab model described in Sect. 5.1. Some of the strongest absorption features are labelled.

best-fit continuum model obtained from the EPIC-pn. All the continuum parameters except the power-law photon index were left free. The photon index was let free only after an overall good fit was obtained. This helps prevent being caught in false $\chi_{v}^{2}$ minima and obtaining a photon index incompatible with the EPIC-pn during the $\chi_{v}^{2}$ minimisation. This method of fixing the power-law photon index until close to the end of the fitting is also used in Steenbrugge et al. (2003).
Initially all the ions in the slab component have negligible column densities. We fitted the column density of each ion one by one, starting from the ions responsible for the strongest absorption lines in the spectrum. All the ions in a slab component have the same flow and rms (broadening) velocities. So after fitting the column densities of all the ions, we decoupled the velocities of some of the ions by creating a separate slab component for each ion. Table 2 shows the velocities determined for these ions. Only decoupling the velocities of the ions with strong absorption lines improves the fit. Decoupling velocities of the ions with weak lines does not improve the fit because large errors are associated with these ions. The ions with weak lines, which do not appear in Table 2, are modelled as one slab component with an outflow velocity of $900 \pm 100 \mathrm{~km} \mathrm{~s}^{-1}$ and rms velocity of $900 \pm 100 \mathrm{~km} \mathrm{~s}^{-1}$.

We only used the slab model to get some understanding of the scale of velocity and column density of the ions, especially those with the clearest absorption lines in the RGS spectrum (e.g. O VIII). To analyse the warm absorber in detail, we used a more realistic model described in the following subsection.

\subsection{Spectral fit using a photoionised absorption model}

The xabs model in SPEX calculates the transmission through a slab of material, where all ionic column densities are linked in a physically consistent fashion through a grid of the XSTAR (Bautista \& Kallman 2001) photoionisation models. We used the default $x a b s$ model where the Spectral Energy Distribution (SED) is based on the one from NGC 5548 as used in Steenbrugge et al. (2005). This is a good approximation for NGC 3516 because both Seyfert 1.5 AGN have very similar SEDs as shown in the NASA/IPAC Extragalactic Database (NED). The advantage of the $x a b s$ model over the slab model is that all relevant ions are taken into account, including those that would be detected only with marginal significance or not detected at all with the slab model (Kaastra et al. 2009).

We started with the EPIC-pn best-fit continuum and Fe K $\alpha$ model established in Sect. 4, leaving the power-law, $m b b$ and $\mathrm{Fe} \mathrm{K} \alpha$ parameters free (except for the power-law photon index, which was kept fixed to the value given in Table 1 until close to the end of the fitting procedure). We applied the $x a b s$ model to the continuum to fit the ionisation parameter $(\xi)$, the hydrogen column density $\left(N_{\mathrm{H}}\right)$, the flow and rms velocities in the EPIC-pn and RGS spectra of Obs. 2 simultaneously. The elemental abundances were fixed at the proto-solar values of Lodders (2003). Because we were fitting EPIC-pn, RGS1 and RGS2 spectra simultaneously, we allowed the relative normalisations of the EPIC-pn and the two RGS instruments to be free parameters. We fixed the instrumental normalisation of RGS1 to 1 and freed the normalisation of the RGS2 and EPIC-pn 
Table 2. Flow and rms (broadening) velocities of the ions with the strongest absorption lines in the Obs. 2 RGS spectrum, determined from the slab model best fit.

\begin{tabular}{llll}
\hline \hline Ion & Flow $v^{a}$ & Rms $v^{a}$ & Observed transitions \\
\hline C VI & $-800 \pm 100$ & $300 \pm 100$ & Ly- $\alpha$, Ly- $\beta$, Ly- $\gamma$ \\
N VI & $-700 \pm 300$ & $600 \pm 200$ & He $\alpha$, He $-\beta$, He- $\gamma$ \\
N VII & $-800 \pm 300$ & $900 \pm 300$ & Ly- $\alpha$, Ly- $\beta$, Ly- $\gamma$ \\
O VII & $-900 \pm 100$ & $300 \pm 100$ & He- $\alpha$, He $-\beta$, He- $\gamma$, He- $\delta$ \\
O VIII & $-1000 \pm 100$ & $370 \pm 40$ & Ly- $\alpha$, Ly $-\beta$, Ly $-\gamma$, Ly $-\delta$ \\
\hline
\end{tabular}

Notes. ${ }^{(a)} \mathrm{km} \mathrm{s}^{-1}$.

instruments. This way problems with differences in relative normalisations between different instruments are avoided (Blustin et al. 2003).

We then included one $x a b s$ component in the model. This corresponds to phase B in Table 4, where the phases are sorted by increasing value of $\xi$. Adding the first xabs component improved $\chi_{v}^{2}$ from 25.3 (2801 d.o.f.) to 3.91 (2797 d.o.f.), by fitting $\xi, N_{\mathrm{H}}$, flow and rms velocities. Our model still required more absorption at $\sim 11-15 \AA$ (expected to be due to hot iron, Fe XVII-Fe XXIV) and $\sim 16-17 \AA$ (most likely due to cold iron, Fe I-Fe XIV). To produce a better fit to the data, we accordingly introduced a second $x a b s$ component to our model. This corresponds to phase $\mathrm{C}$ in Table 4 . The addition of phase $\mathrm{C}$, which is the one with the highest ionisation parameter, further improved $\chi_{v}^{2}$ from 3.91 (2797 d.o.f.) to 1.88 (2793 d.o.f.). We found that after adding two xabs components, absorption by iron, especially the M-shell iron (Fe I-Fe XIV) forming the Unresolved Transition Array (UTA) at 16-17 $\AA$ (Behar et al. 2001), was still not properly modelled. To model the UTA, a phase with an ionisation lower than the two previously identified was required. Therefore, a third $x a b s$ component (phase A) was added to our model. The addition of phase A improved $\chi_{v}^{2}$ from 1.88 (2793 d.o.f.) to 1.63 (2789 d.o.f.) as the UTA was fitted well. At this point, the power-law photon index $(\Gamma)$ was left free to vary in the fitting procedure for Obs. 2, but neither the value of $\Gamma$ (1.82) nor $\chi_{v}^{2}$ changed significantly.

To attempt to further improve the quality of our fit, we freed the abundances of only those elements with the strongest absorption features in the RGS spectrum: $\mathrm{N}, \mathrm{O}$ and Fe. In order not to increase the number of free parameters unnecessarily, we coupled the abundances of all three phases. The precise abundances of elements in each phase cannot be accurately determined, because letting them all free results in some cases in unphysical values. Assuming that different phases have the same abundances is already a more progressive approach than the alternative of assuming cosmic abundances for all the phases. The abundance coupling approach is also used in Kaastra et al. (2003), where they fixed the abundances of a cool gas component to those of a hot one to reduce the number of free parameters. Freeing the N, O and Fe abundances produced a better fit, because $\chi_{v}^{2}$ fell from 1.63 (2788 d.o.f.) to 1.52 (2785 d.o.f.).

One of the ionisation phases in Turner et al. (2008) with $\log \xi$ of 2.2 has a partial covering fraction. Therefore we tested at this stage of the fitting process whether any of our three phases was partially covering the source. We freed the covering fraction (fcov) of the three phases from the default value of 1 . The covering fraction of one of the phases (Phase B with $\log \xi$ of 2.4) was found to change from 1 to 0.55 , while the fcov of the other two phases remained practically unchanged. By freeing the covering fraction of phase $\mathrm{B}, \chi_{v}^{2}$ fell from 1.52 (2785 d.o.f.)
Table 3. Best-fit parameters of the continuum, obtained from the three-phase $x a b s$ model simultaneous fit to the EPIC-pn, RGS1 and RGS2 spectra.

\begin{tabular}{ccccc}
\hline \hline \multicolumn{2}{c}{ Power-law: } & \multicolumn{2}{c}{ Modified black body: $^{c}$} \\
Obs. photon index $(\Gamma)$ normalisation ${ }^{a}$ & temperature $^{b}$ & normalisation $^{c}$ \\
\hline 1 & $1.85 \pm 0.02$ & $3.3 \pm 0.1$ & $189 \pm 2$ & $1.3 \pm 0.1$ \\
2 & $1.82 \pm 0.01$ & $2.8 \pm 0.1$ & $186 \pm 3$ & $1.5 \pm 0.1$ \\
3 & $1.70 \pm 0.02$ & $1.9 \pm 0.1$ & $211 \pm 4$ & $0.5 \pm 0.1$ \\
4 & $1.84 \pm 0.02$ & $2.9 \pm 0.1$ & $189 \pm 2$ & $1.4 \pm 0.1$ \\
\hline
\end{tabular}

Notes. ${ }^{(a)} 10^{51}$ photons $\mathrm{s}^{-1} \mathrm{keV}^{-1}$ at $1 \mathrm{keV}$; ${ }^{(b)} \mathrm{eV}$; ${ }^{(c)}$ emitting area times square root of the electron density in $10^{33} \mathrm{~cm}^{0.5}$.

to 1.44 (2784 d.o.f.). Indeed there are absorption lines in the 11-13 $\AA$ region of the RGS spectrum that are not fitted well if fcov is fixed to 1, as well as the UTA and the O VII He- $\alpha$ line. This made a partial covering of the continuum necessary for phase B, but not for the other two phases. Consequently below we allowed phase $\mathrm{B}$ to have a partial covering fraction, and the fcov of phases $\mathrm{A}$ and $\mathrm{C}$ were fixed to 1 . The best-fit parameters for Obs. 2 (power-law, $m b b$, xabs and abundances) are listed in Tables 3-5. A plot of the whole RGS spectrum, the EPIC-pn data and the best-fit model for Obs. 2 is shown in Fig. 5. In Fig. 6, close-ups of the RGS spectrum and the best-fit model are presented.

Having obtained the $x a b s$ model best-fit for Obs. 2, we used it as the starting point for each of the other observations, leaving all the parameters quoted so far free, with the exception of the abundances of the xabs phases, which were fixed at the Obs. 2 best-fit (the abundance values are shown in Table 5). The bestfit parameters of the continuum and ionisation phases of Obs. 1, Obs. 3 and Obs. 4, modelled with the $x a b s$ photoionised absorption, are also shown in Tables 3 and 4 . Note that the $\chi_{v}^{2}$ values in Table 4 are further improved by modelling the observed emission features discussed in Sect. 5.5. In the following subsection (Sect. 5.3), we describe how the Obs. 2 best-fit was applied to the Obs. 3 spectrum to investigate whether the covering fraction of phase $B$ is variable or not.

\subsection{Spectral variability in Obs. 3}

As shown in Table 4, phase B in Obs. 2 has a best-fit covering fraction $(f c o v)$ of 0.55 . Our phase B is very similar to the partially covering phase (zone 3 ) of Turner et al. (2008) in terms of the ionisation parameter. We found a $\log \xi \sim 2.4$ versus Turner et al.'s $\log \xi \sim 2.2$. However, the column density of our phase B $\left(N_{\mathrm{H}} \sim 2.0 \times 10^{22} \mathrm{~cm}^{-2}\right)$ is about a factor of 10 smaller than that in Turner et al. (2008) $\left(N_{\mathrm{H}} \sim 20 \times 10^{22} \mathrm{~cm}^{-2}\right)$. In Sect. 6.2, we discuss the values of $N_{\mathrm{H}}$ of warm absorber phases in NGC 3516 found by different authors.

Turner et al. (2008) explained the dip in the X-ray lightcurve of Obs. 3 by varying only the covering fraction of their zone 3. In order to try and reproduce their results, we applied our Obs. 2 best fit (described in Sect. 5.2) to Obs. 3 by keeping all the parameters fixed and freeing only the covering fraction. As for Obs. 2, we fitted the EPIC-pn and RGS spectra simultaneously. Applying the Obs. 2 best fit to Obs. 3 we obtained an initial $\chi_{v}^{2}$ of 225 (2711 d.o.f.). We found that by freeing only the covering fraction no good fit was achieved; the covering fraction goes to 1 and $a \chi_{v}^{2}$ of 130 (2710 d.o.f.) is obtained. On the other hand, when we freed only the continuum parameters, a much better fit was obtained, $\chi_{v}^{2}$ of 1.76 (2707 d.o.f.). Figures 7 and 8 depict the 
A\&A 514, A100 (2010)

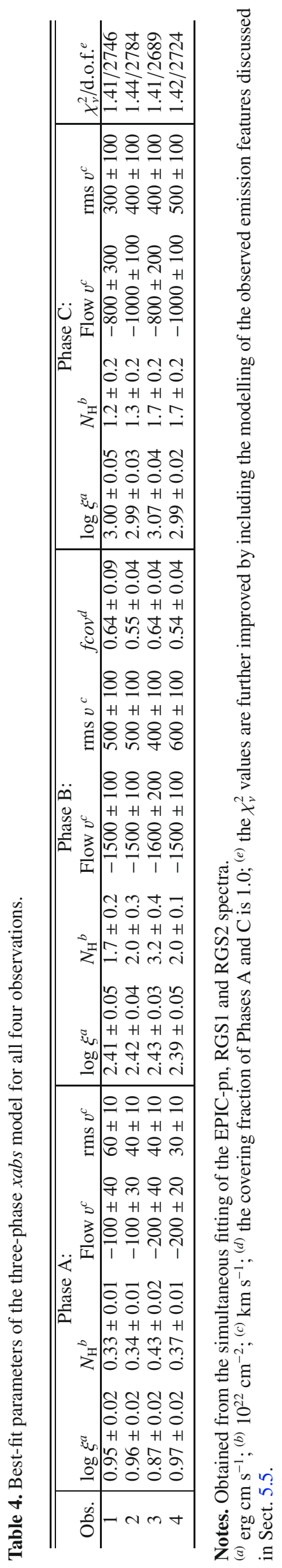

Table 5. The elemental abundances of the three-phase xabs model of Obs. 2 fitted simultaneously to the EPIC-pn, RGS1 and RGS2 spectra.

\begin{tabular}{cc}
\hline \hline Element & Abundance relative to $\mathrm{H}$ \\
\hline $\mathrm{N}$ & $1.5 \pm 0.1$ \\
$\mathrm{O}$ & $1.4 \pm 0.1$ \\
$\mathrm{Fe}$ & $1.8 \pm 0.1$ \\
Other elements & 1 (f) \\
\hline
\end{tabular}

Notes. The abundances of the three phases were coupled to each other in the fits. All the abundances are relative to the proto-solar model of Lodders (2003). The reference element is $\mathrm{H}$.

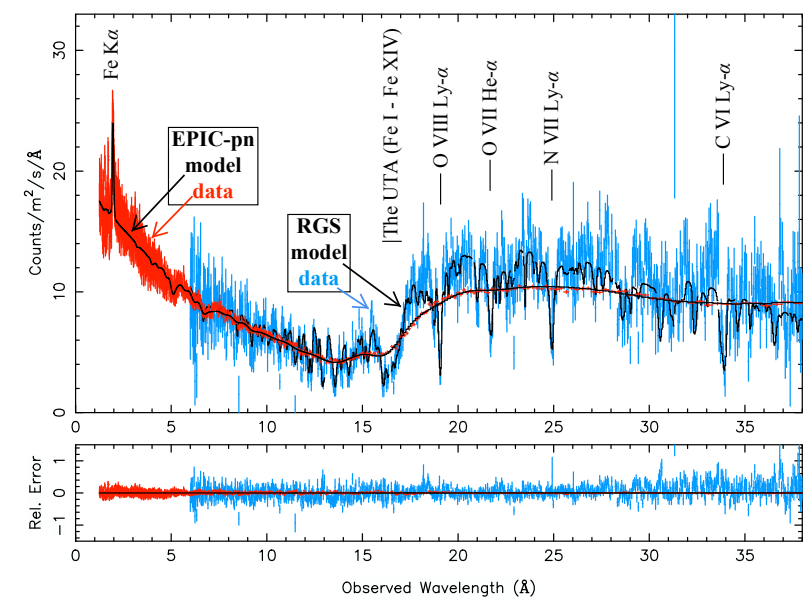

Fig. 5. Observation 2 best-fit xabs model, fitted simultaneously to the RGS and EPIC-pn spectra. The RGS data are shown in blue, the EPIC-pn data in red and the models in black. Close-ups of the RGS spectrum, showing the spectral features more clearly, are presented in Fig. 6. Note that both RGS and EPIC-pn spectra in this figure have been divided by the instrument's effective area, thus are plotted on the same scale.

difference between the two approaches to Obs. 3, which proves that changing the continuum parameters is a much more feasible solution than changing only the covering fraction of phase B in order to fit the Obs. 3 spectrum.

We then tried freeing both the continuum and covering fraction. In this case, the covering fraction becomes 0.70 and $\chi_{v}^{2}$ goes to 1.65 (2706 d.o.f.). We then freed the ionisation parameter and $N_{\mathrm{H}}$ of the three $x a b s$ phases, parameters of the Fe K $\alpha$ line and the relative normalisations of the EPIC-pn and RGS instruments; the $\chi_{v}^{2}$ improved to 1.42 (2695 d.o.f.). Finally, we freed the velocities of the $x a b s$ phases, keeping the abundances fixed. In this case, the fit does not improve significantly, a $\chi_{v}^{2}$ of 1.41 (2689 d.o.f.) was obtained. The best-fit results for Obs. 3, as for the other three observations, are shown in Tables 3 and 4.

It is worth noting the advantage of modelling both the RGS and EPIC-pn together, which leads to simultaneously constraining the broadband continuum using the EPIC-pn and modelling the absorption features with the RGS. This approach has a drawback because due to smaller statistical fractional errors associated with the EPIC-pn compared to the RGS, the best fit favours the EPIC-pn. But by making sure that the RGS absorption features are fitted properly, this weakness is not a problem.

\subsection{The partially covering phase $B$}

Figure 9 shows how each one of the three phases in our warm absorber model contributes to the best fit for the four observations. 

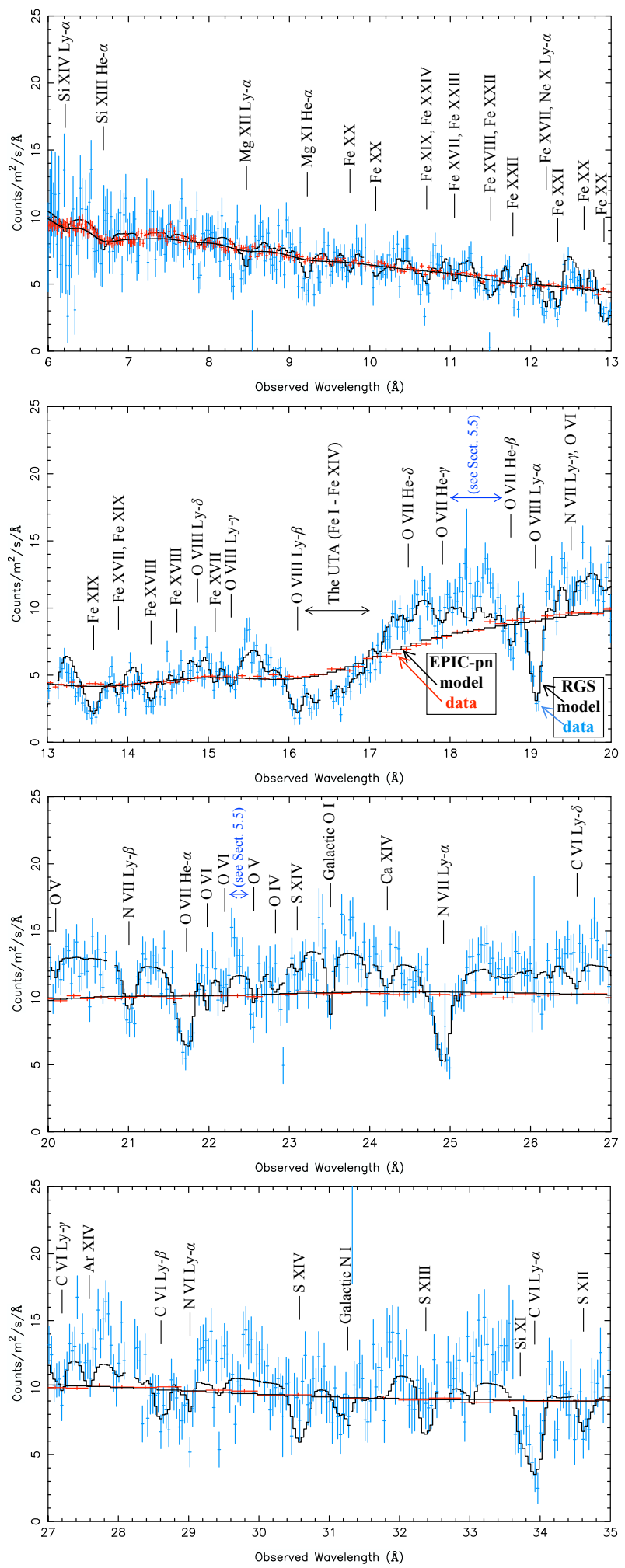

Fig. 6. Observation 2 best fit $x a b s$ model, fitted simultaneously to the RGS and EPIC-pn spectra. The RGS data are shown in blue, the EPIC-pn data in red and the models in black.

Phases A, B and C are plotted in black, blue and red, respectively. Phase $\mathrm{A}$, which is the lowest ionisation phase, is associated with M-shell Fe absorption forming the UTA (Behar et al. 2001) between 16 and $17 \AA$. Phase $C$, the highest ionisation phase, is associated with highly ionised Fe. Phase B, of intermediate ionisation, is the phase with the partial covering fraction.
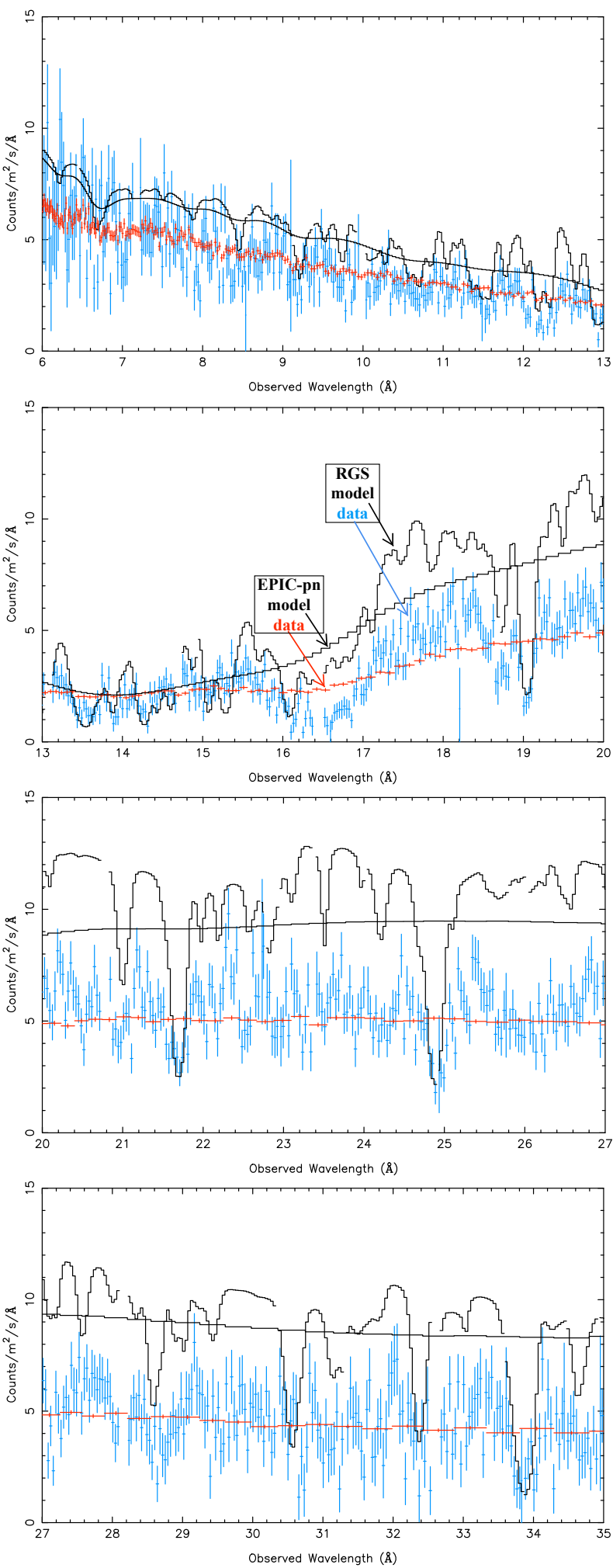

Fig. 7. Simultaneous fit made to the EPIC-pn and RGS spectra of Obs. 3 with the best-fit model of Obs. 2 and fitting only the covering fraction of phase B. The $\chi_{v}^{2}$ is 130 (2710 d.o.f.). The RGS data are shown in blue, the EPIC-pn data in red and the models in black.

In the previous subsection (Sect. 5.3), we showed that by changing only the covering fraction of phase $\mathrm{B}$, one cannot fit the Obs. 3 spectrum. To confirm that fcov of phase B does not change significantly between observations, we examined our model closely to look for absorption lines unique to phase B 

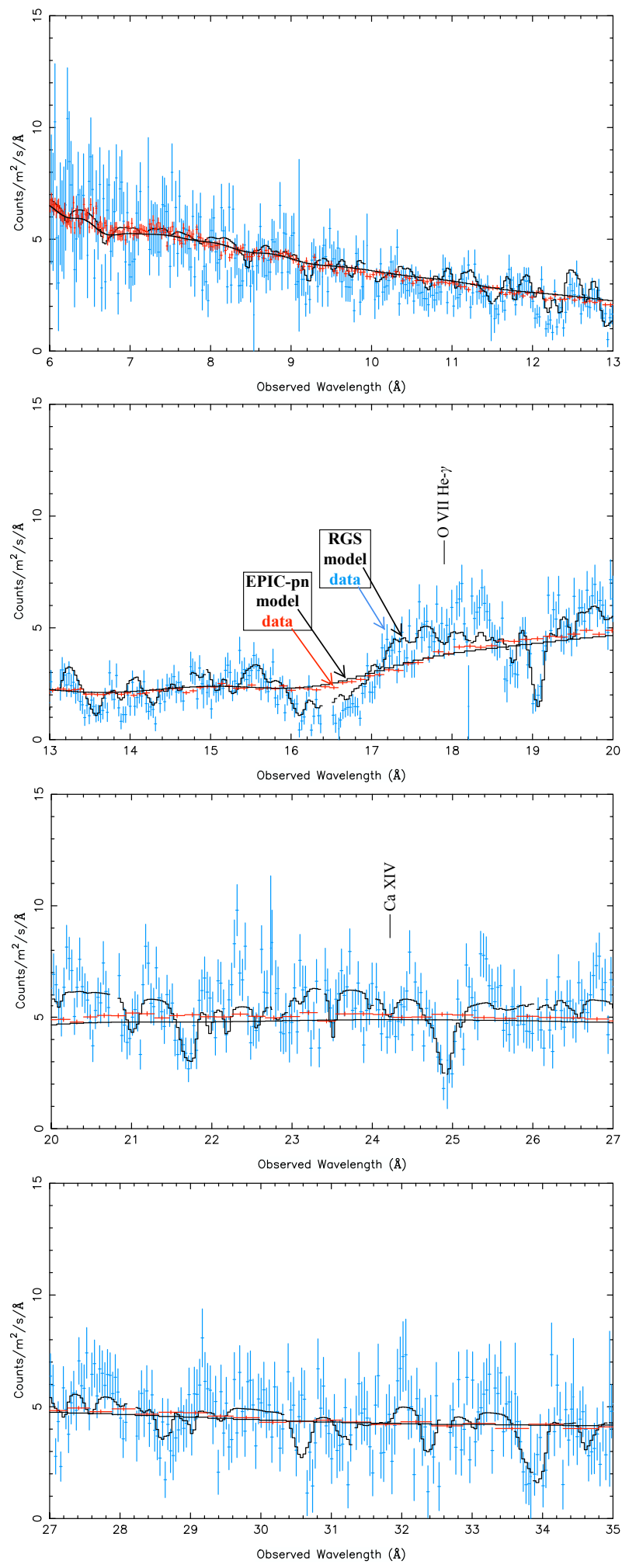

Fig. 8. Simultaneous fit made to the EPIC-pn and RGS spectra of Obs. 3 with the best-fit model of Obs. 2 and fitting only the continuum parameters (maintaining $f$ cov $=0.55$ ). The $\chi_{v}^{2}$ is 1.76 (2707 d.o.f.). The RGS data are shown in blue, the EPIC-pn data in red and the models in black. The significance of the O VII He- $\gamma$ and Ca XIV lines is discussed in Sect. 5.4.

(i.e. that do not appear in phases A and C) and to see if they change between Obs. 2 and Obs. 3. This is important because by observing a change in the depth of these lines, we can investigate
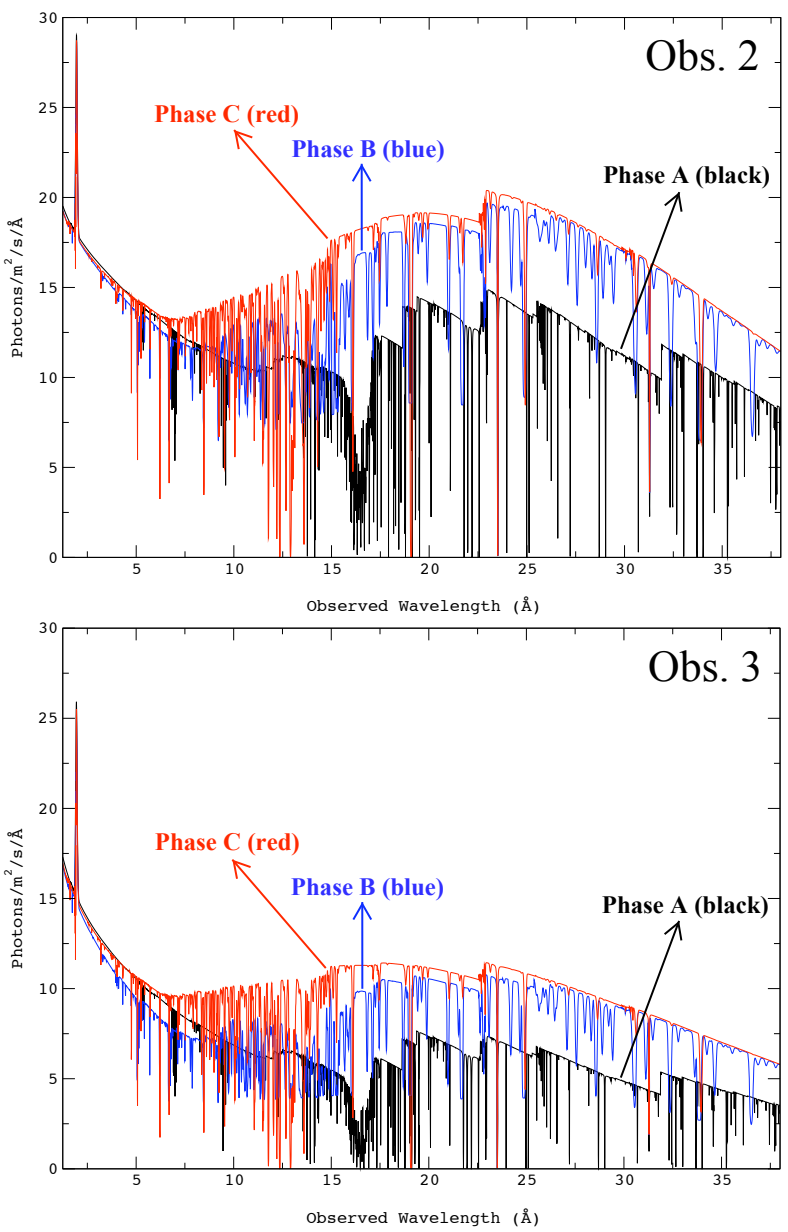

Fig. 9. Our best-fit warm absorber xabs model showing the three phases applied separately to the continuum (phase A: black, phase B: blue, phase C: red) from simultaneous fitting of the EPIC-pn and RGS spectra of Obs. 2 (top) and Obs. 3 (bottom). The best-fit parameters of the models are given in Tables 3-5. The models for Obs. 1 and Obs. 4 look identical to that of Obs. 2 (top).

the effect of a covering fraction change. We found three such absorption lines, which are only modelled by phase B. They are O VII He- $\gamma(17.8 \AA)$, Ca XIV (24.1 $)$ ) and S XII (36.4 $\AA$ ). Figure 10 shows the O VII He- $\gamma$ and Ca XIV lines in our model; the S XII line is not displayed separately to save space, but the following is also true for the S XII line. Comparing the fits in Figs. 6 (Obs. 2) and 8 (Obs. 3), we can see that the O VII He- $\gamma$ and Ca XIV are fitted well in both. It is important to note that in both figures the covering fraction value is the same (i.e. 0.55). If the covering fraction of phase $\mathrm{B}$ had changed significantly in Obs. 3, we would have expected the O VII He- $\gamma$ and Ca XIV lines in Fig. 8 not to be fully fitted. The fact that they are fitted well by varying only the continuum parameters is an extra piece of evidence that the variability seen in the lightcurve is mostly intrinsic to the source, and not the warm absorber.

\subsection{Narrow and broad emission features}

The only significant narrow emission feature in the RGS spectra of the four observations of NGC 3516 is the O VII forbidden line (rest frame wavelength of $22.10 \AA$ ). Figure 11 shows the Obs. 2 and Obs. 3 spectra and best fit around the O VII triplet region. This line was modelled with a Gaussian profile (Gaus), and its best-fit parameters are given in Table 6. The RGS spectrum 


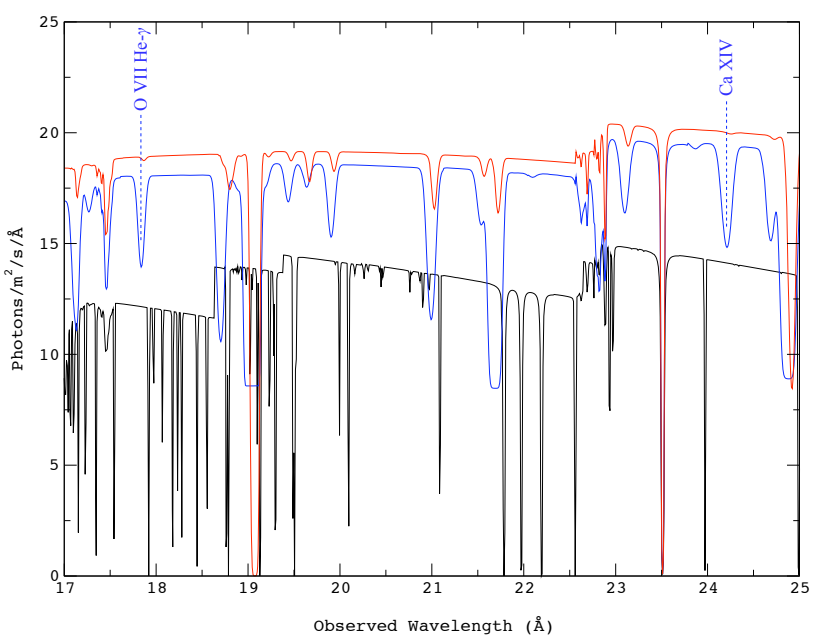

Fig. 10. Observation 2 best-fit xabs model, showing that the O VII He- $\gamma$ and Ca XIV absorption lines are only modelled in phase B. Phase A is shown in black, phase $\mathrm{B}$ in blue and phase $\mathrm{C}$ in red.

of NGC 3516 around the O VII triplet is very similar to that of NGC 5548 presented by Steenbrugge et al. (2003): in that case, the forbidden line is present in emission and outflowing at around $110 \mathrm{~km} \mathrm{~s}^{-1}$, the intercombination line is absent or very weak and the resonance line is seen in absorption; there are also absorption lines by O V and O VI. As suggested by Steenbrugge et al. (2003), one possible explanation for not detecting O VII intercombination emission is blending of the intercombination line with the O VI absorption line.

From Figs. 6 and 8 an excess of emission is noticeable in the RGS data at around 18.0 to $18.5 \AA$. This excess is observed in all four observations. Also, in this region of the spectrum, phase A, the lowest-ionised phase, produces absorption by O VI. We first fitted the excess in Obs. 2 with a simple Gaussian profile; best-fit line wavelength of $18.16 \pm 0.04 \AA$ (in the rest frame of the AGN) and $F W H M$ of $0.47 \pm 0.09 \AA\left(\sigma_{v}=3300 \pm 600 \mathrm{~km} \mathrm{~s}^{-1}\right)$ were obtained with a $\chi_{v}^{2}$ of 1.40 (2781 d.o.f.). The wavelength of the emission line does not correspond to any prominent transition, unless this is emission by O VIII Ly- $\alpha$ (rest-frame $\lambda$ of $18.97 \AA$ ) with an outflow velocity of about $13000 \mathrm{~km} \mathrm{~s}^{-1}$. Therefore we investigated as an alternative whether the excess could be caused by a relativistically-broadened O VIII accretion disk emission line. If emission from the inner disk is influenced by general relativistic effects, the lines would appear to be broad and asymmetric. These lines were first proposed to explain the spectra of the Narrow Line Seyfert 1 galaxies MCG -6-30-15 and Mrk 766 (Branduardi-Raymont et al. 2001). We added a narrow emission line (delt component in SPEX) at the rest frame wavelength of the O VIII Ly- $\alpha$ line (18.97 $\AA$ ) at the redshift of NGC 3516, and convolved it with the relativistic disk line profile of Laor (1991) (laor). Parameters of the laor component are the disk inner and outer radii $r_{\text {in }}$ and $r_{\text {out }}$, its inclination angle $i$ and the emissivity index $q$, which appears in the emissivity law and is taken to be proportional to $\left(r^{2}+h^{2}\right)^{-q / 2}$, where $r$ is the radius in the accretion disk and $h$ a scale height (Kaastra et al. 2002). By adding the relativistic $\mathrm{O}$ VIII line to the $x a b s$ best-fit model of Obs. 2, our fit improved as $\chi_{v}^{2}$ fell from 1.44 (2784 d.o.f.) to 1.40 (2779 d.o.f.) and the excess was well fitted. The line profile and the best fit are shown in Fig. 12.

The inner disk radius is found to be $14 \pm 3 G M / c^{2}$ and the outer disk radius is poorly constrained. The signal of the red tail of the line is not strong enough to be modelled accurately;
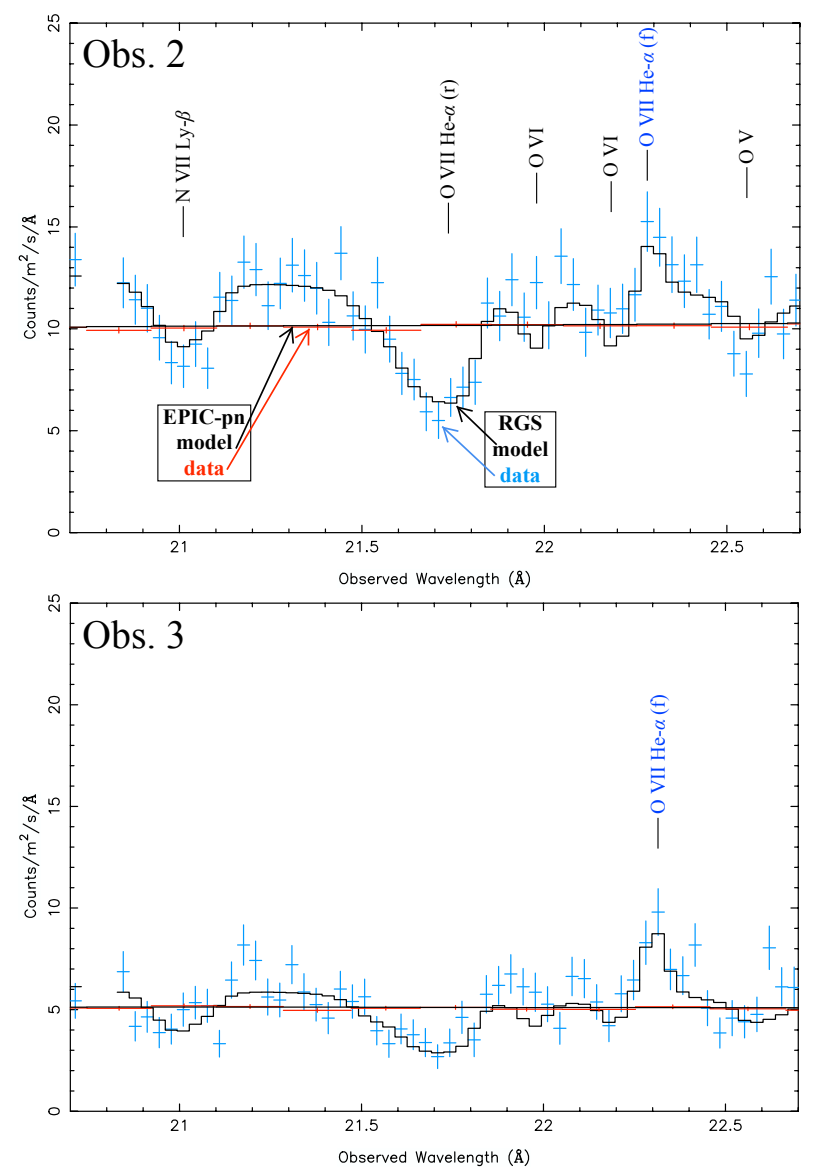

Fig. 11. Presence of forbidden O VII line emission in Obs. 2 (top) and Obs. 3 (bottom). The RGS data are shown in blue, the EPIC-pn data in red and the models in black.

therefore, as the asymmetry of the line is not very clear in the data, the broadened Gaussian and the Laor profile fit the excess equally well in our modelling; however, as explained earlier, a Gaussian profile does not correspond to any prominent transition.

The disk inclination angle is constrained very well in our model for all four observations; this is because its value strongly depends on the position of the bulk of the excess emission in the spectrum: a higher inclination angle causes the emission line profile, shown in Fig. 12 (bottom), to move to lower energies and vice versa. As the excess emission is clear with respect to the continuum, the disk inclination angle is derived to be $33^{\circ} \pm 2$.

\section{Discussion}

In this work we demonstrated the advantage of combining RGS high resolution with EPIC-pn spectroscopy in modelling an AGN warm absorber. EPIC-pn constrains the continuum well, but has insufficient resolution to resolve absorption and emission features seen in the warm absorber (e.g. the Fe UTA and O VIII Ly- $\alpha$ absorption as well as the O VII He- $\alpha$ (f) emission line shown in Figs. 6 and 11). Therefore, to investigate changes in the warm absorber between observations, careful examination and fitting of the RGS spectrum are needed alongside fitting the EPIC-pn spectrum. In the subsections below we discuss our results and their physical implications. 
A\&A 514, A100 (2010)

Table 6. The best-fit parameters of the narrow O VII forbidden emission line shown in Fig. 11.

\begin{tabular}{lllll}
\hline \hline Parameter & Obs. 1 & Obs. 2 & Obs. 3 & Obs. 4 \\
\hline$\lambda_{0}{ }^{a}$ & 22.10 & 22.10 & 22.10 & 22.10 \\
$\lambda^{b}$ & $22.11 \pm 0.06$ & $22.09 \pm 0.04$ & $22.11 \pm 0.02$ & $22.12 \pm 0.08$ \\
Flow $v^{c}$ & $+100 \pm 800$ & $-100 \pm 500$ & $+100 \pm 300$ & $+300 \pm 1100$ \\
$F W H M^{d}$ & $<0.1$ & $<0.1$ & $<0.1$ & $<0.1$ \\
$\sigma_{v}{ }^{e}$ & $<600$ & $<600$ & $<600$ & $<600$ \\
Line Normalisation $^{f}$ & $1.0 \pm 0.6$ & $1.0 \pm 0.6$ & $1.7 \pm 0.5$ & $0.9 \pm 0.7$ \\
\hline
\end{tabular}

Notes. Obtained by adding a Gaussian component to the best-fit xabs model of all four observations. The errors correspond to a $\Delta \chi^{2}$ of 2 .

(a) Theoretical rest frame wavelength in $\AA$; ${ }^{(b)}$ wavelength in the rest frame of NGC 3516 in $\AA ;{ }^{(c)} \mathrm{km} \mathrm{s}^{-1} ;{ }^{(d)} \AA$; ${ }^{(e)} \mathrm{rms}$ velocity in $\mathrm{km} \mathrm{s}{ }^{-1}\left(\sigma_{v}=\right.$ FWHM $/ \sqrt{\ln 256}) ;(f) 10^{49}$ photons s ${ }^{-1}$.
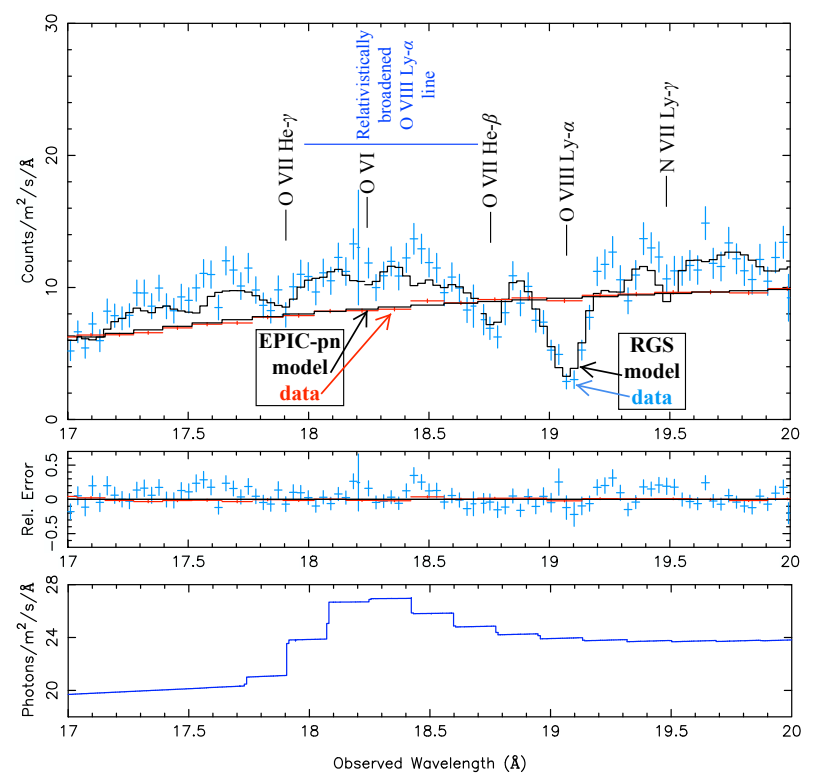

Fig. 12. Top panel: the Obs. 2 best-fit xabsmodel fitted simultaneously to the RGS and EPIC-pn spectra with the relativistic emission line included (compare with Fig. 6, where the line is not included). The RGS data are shown in blue, the EPIC-pn data in red and the models in black. Middle panel: residuals of the fit in the top panel. Bottom panel: the Obs. 2 best-fit model of the relativistic O VIII Ly- $\alpha$ emission line added to the continuum. The model is shown in the observed frame, and for clarity of presentation no absorption component of our model is included in this figure.

\subsection{The Fe Ko line}

We modelled the broad $\mathrm{Fe} \mathrm{K} \alpha$ line at $\sim 6.4 \mathrm{keV}$ with a simple Gaussian profile. For an in-depth spectral analysis of the $\mathrm{Fe} \mathrm{K}$ regime, we refer the reader to Turner et al. (2008) in which the Chandra HETG spectrum is used to model the $\mathrm{Fe} \mathrm{K} \alpha$, $\mathrm{Fe} \mathrm{K} \beta$ and Fe XXVI emission lines in detail. However, from our simple modelling of the line, described in Sect. 4 (Table 1), we find that the Fe $\mathrm{K} \alpha$ flux remains practically unchanged between observations, whereas the power-law flux falls significantly in Obs. 3. It is known that the variability of the $\mathrm{Fe} \mathrm{K} \alpha$ line in general is not correlated to that of the observed continuum in a trivial manner (Miniutti \& Fabian 2004). The iron line does not always respond to variations in the continuum: in some cases the $\mathrm{Fe} \mathrm{K} \alpha$ line can appear to be constant while the continuum varies by a large amplitude (e.g. Markowitz et al. 2003). Using the EPIC-pn spectra, we cannot examine the $\mathrm{Fe} \mathrm{K} \alpha$ profile as accurately as with the Chandra HETG, so we will not discuss this any further.

\subsection{The warm absorber structure}

We found that three phases of ionisation are adequate to model the warm absorption in NGC 3516. The elemental abundances are unlikely to be different in the three phases, because it is possible to fit the spectrum very well by coupling all the abundances at values close to solar (Table 5). There is also no need for a large over-abundance of iron to fit the UTA.

A comparison of $\xi, N_{\mathrm{H}}$ and outflow velocity values of warm absorber phases from recent observations of NGC 3516 reported by different authors is shown in Table 7 . The ionisation parameters of our phases $\mathrm{B}$ and $\mathrm{C}$ are remarkably similar to those found by Turner et al. (2005) during the 2001 observations. We found no need for an absorption by a fourth phase (identified by Chandra HETG to correspond to $\log \xi \sim 4.3$ in Turner et al. 2008) from our simultaneous EPIC-pn and RGS fittings, which are not very sensitive to this high ionisation gas. Note that $\log \xi$ values are not strictly comparable as they are SED dependent, although the differences are likely to be only of the order of some tenth of dex. The outflow velocity of our partially covering phase B is practically the same as that in Turner et al. (2008) (their partially covering Zone 3); these phases also have very similar ionisation parameters, suggesting they represent the same photoionised gas. The second phase in Turner et al. (2005), which was also partially covering, is again very similar to our phase B in terms of ionisation parameter and outflow velocity (albeit this is smaller in 2005). The pattern that emerges by comparing the outflow velocities of all the phases found by different authors is the existence of low-ionised phases with a very low outflow velocity and higher ionisation phases with outflow velocities between $1000 \mathrm{~km} \mathrm{~s}^{-1}$ and $1500 \mathrm{~km} \mathrm{~s}^{-1}$.

The partially covering phase is the phase with the highest outflow velocity and most likely closest to the central engine. Since we do not find changes in the occultation of the nuclear source, there is no evidence of a transverse component of velocity for the phase. The minimum distance of this phase from the central engine can be estimated assuming the outflow velocity we measure to be greater than or equal to the escape velocity $v_{\text {esc }}=\sqrt{2 G M / r}$, where $G$ is the gravitational constant, $M$ the black hole mass and $r$ the distance of the absorber phase from the black hole. Since the partially covering phase B is outflowing at $1500 \mathrm{~km} \mathrm{~s}^{-1}$, using the NGC 3516 black hole mass estimate of $2.95 \times 10^{7} M_{\odot}$ (Nikołajuk et al. 2006), we obtain $r \gtrsim 0.1 \mathrm{pc}$. Using the expression for the ionisation parameter definition (Eq. (1)) and taking $L_{\text {ion }} \simeq 3.85 \times 10^{43} \mathrm{erg} \mathrm{s}^{-1}$ (derived from the fit of Obs. 2) we find the hydrogen number density $n \lesssim 1.3 \times 10^{8} \mathrm{~cm}^{-3}$. Combining this with the relation $N_{\mathrm{H}} \sim n \Delta r$, where $\Delta r$ is the thickness of the absorber phase, we find $\Delta r \gtrsim 5 \times 10^{-5} \mathrm{pc}$. This suggests a thin spherical shell of gas, which is a feasible scenario for partial covering. 
Table 7. Comparison of the ionisation parameters, hydrogen column densities and outflow velocities of the NGC 3516 warm absorber phases found in recent observations, shown in increasing order of $\xi$.

\begin{tabular}{|c|c|c|c|c|c|c|c|c|c|c|c|c|c|}
\hline \multicolumn{3}{|c|}{ 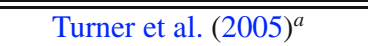 } & \multicolumn{3}{|c|}{ "Markowitz et al. $(2008)^{b}$} & \multicolumn{4}{|c|}{ 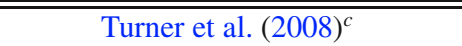 } & \multicolumn{4}{|c|}{ Present work $^{d}$} \\
\hline $\log \xi^{e} \sim$ & $N_{\mathrm{H}}^{f} \sim$ & $v^{g} \sim$ & $\log \xi^{e} \sim$ & $N_{\mathrm{H}}^{f} \sim$ & $v^{g} \sim$ & $\log \xi^{e} \sim$ & $N_{\mathrm{H}}{ }^{f} \sim$ & $v^{g} \sim$ & zone & $\log \xi^{e} \sim$ & $N_{\mathrm{H}}^{f} \sim$ & $v^{g} \sim$ & phase \\
\hline- & - & - & - & - & - & -2.4 & 0.2 & $?$ & 1 & - & - & - & - \\
\hline-0.5 & 0.5 & -200 & 0.3 & 5.5 & $?$ & 0.25 & 0.05 & ? & 2 & 0.9 & 0.4 & -100 & A \\
\hline 2.5 & 25 & -1100 & - & - & - & 2.2 & 20 & -1600 & 3 & 2.4 & 2 & -1500 & $\mathrm{~B}$ \\
\hline 3.0 & 2 & -1100 & 3.7 & 4 & $-1100^{h}$ & 4.3 & 26 & -1000 & 4 & 3.0 & 1 & -900 & $\mathrm{C}$ \\
\hline
\end{tabular}

Notes. ${ }^{(a)}$ From the Apr. and Nov. 2001 XMM-Newton EPIC-pn spectra. The values of the ionisation parameter in $\xi$ form are given in Turner et al. (2008); ${ }^{(b)}$ from the Oct. 2005 Suzaku XIS and HXD spectra; ${ }^{(c)}$ from the Oct. 2006 XMM-Newton EPIC-pn and Chandra HETG spectra; ${ }^{(d)}$ from the Oct. 2006 XMM-Newton EPIC-pn and RGS spectra; ${ }^{(e)} \mathrm{erg} \mathrm{cm} \mathrm{s}{ }^{-1}{ }^{(f)} 10^{22} \mathrm{~cm}^{-2}$; ${ }^{(g)}$ flow velocity in $\mathrm{km} \mathrm{s}^{-1}$; ${ }^{(h)}$ based on the velocity obtained in Turner et al. (2005).

The column densities $N_{\mathrm{H}}$ of all three phases of our warm absorber cover a relatively small range: $\sim 0.4-2 \times 10^{22} \mathrm{~cm}^{-2}$, and we do not find "heavy" $\left(\gtrsim 20 \times 10^{22} \mathrm{~cm}^{-2}\right)$ absorber phases as in Turner et al. $(2005,2008)$, especially those corresponding to our phase B. Still our range of $N_{\mathrm{H}}$ is consistent with the values obtained from other X-ray observations of NGC 3516: Costantini et al. (2000) found a warm absorber column of $\sim 2-3 \times 10^{22} \mathrm{~cm}^{-2}$ from BeppoSAX spectra; Netzer et al. (2002) found a line-ofsight absorber with $N_{\mathrm{H}}$ of $\sim 1 \times 10^{22} \mathrm{~cm}^{-2}$ from both a 1994 ASCA observation and a 2000 Chandra observation; Markowitz et al. (2008) found their two warm absorber phases to have $N_{\mathrm{H}}$ of $\sim 4$ and $5.5 \times 10^{22} \mathrm{~cm}^{-2}$.

Turner et al. (2005) concluded that their three absorber phases cannot be in thermal equilibrium with each other in NGC 3516. We can consider the photoionisation equilibrium curve technique (S-curve, Krolik et al. 1981) for the SED used in our analysis (NGC 5548, Steenbrugge et al. 2005). The S-curve marks the points of a temperature $T$ versus a pressure form of the ionisation parameter $\Xi$ at which the pressure $P$ is constant. The parameter $\Xi$ is related to $\xi$ by

$\Xi=\frac{L_{\text {ion }}}{4 \pi c r^{2} P}=0.961 \times 10^{4} \frac{\xi}{T}$,

where $L_{\text {ion }}$ is the luminosity of the ionising source, $c$ is the speed of light, $r$ is the distance of the warm absorber phase from the ionising source, $P$ is the pressure and $T$ the temperature. Steenbrugge et al. (2005) found that the S-curve for NGC 5548 has no negative slope anywhere; applying this to NGC 3516, we can conclude that the three absorption phases we identify in NGC 3516 outflowing with different velocities are unlikely to be in pressure equilibrium. However, it is worth noting that different photoionisation codes, variations in the shape of the SED and selecting different energy ranges for the construction of the SED (Smith 2009; Chakravorty et al. 2009) can produce some differences in the S-curve. Because the ionisation parameters, column densities and outflow velocities of our phases $B$ and $C$ are similar (although not in pressure equilibrium), and more importantly the covering fraction of phase B is not variable, phase B could have the same origin as the fully covering phase $\mathrm{C}$. This disfavours a clumpy disk-wind scenario as the most likely explanation for the origin of phase $\mathrm{B}$.

\subsection{Narrow and broad emission features}

The RGS spectrum shows evidence for both narrow and broad emission features. The O VII (f) emission line is found to have a lower flow velocity compared to the observed absorption lines of the high ionisation phases B and C; the flow velocity is in fact consistent with zero and with that of the lowest ionisation phase A. Smith et al. (2008) have observed the O VII (f) line to have a very low outflow velocity compared to other soft X-ray emission lines in Ark 564, although in that case all absorption phases also have flow velocities consistent with zero. This lack of a velocity shift from the rest wavelength agrees with a scenario in which we are observing emission from a fully visible spherically symmetric outflowing shell around the nuclear source, thus no net velocity of O VII will be detected. Another possible explanation is that the O VII line originates in the Narrow Line Region (NLR), where the emission lines are produced in a gas of comparatively small velocity dispersion and low density (see for e.g. Kaastra et al. (2002), where the forbidden O VII line in NGC 5548 is suggested to come from the NLR). As the forbidden line is the most intense line in the $\mathrm{O}$ VII triplet, a photoionised gas with low density is required.

We have found possible evidence for a relativistically broadened emission line of O VIII Ly- $\alpha$. However, the line is weaker than the strong O VIII, N VII and C VI Ly- $\alpha$ lines reported by Branduardi-Raymont et al. (2001) and Sako et al. (2003) in MCG -6-30-15 and Mrk 766. Evidence for weak broadened emission lines of O VIII and N VII Ly- $\alpha$ is also found in NGC 5548 by Kaastra et al. (2002). The disk inclination angle obtained from our fit to the O VIII Ly- $\alpha$ profile is $33^{\circ} \pm 2$. Wu \& Han (2001) and Zhang \& Wu (2002) have calculated the inclination angles of the Broad Line Region (BLR) of several Seyfert 1 galaxies using already published bulge stellar velocity dispersions and black hole masses estimated by reverberation mapping. They calculated an inclination angle of $38.3^{\circ} \pm 7.6$ for NGC 3516, which would imply co-planarity of the BLR with the AGN accretion disk.

\subsection{Intrinsic continuum versus warm absorber variability}

The galaxy NGC 3516 has a history of large amplitude continuum variability between observations. Netzer et al. (2002) reported a large drop in flux (factor of $\sim 50$ at $1 \mathrm{keV}$ ) between an ASCA observation in 1994 and a Chandra observation in 2000. They found that the observed flux and spectral variability at these epochs were consistent with a constant column density of lineof-sight material reacting to changes in the ionising continuum. In the 2006 XMM-Newton data we have found the observed spectral and flux variability to be unrelated to changes in the covering fraction of phase B, unlike what is reported by Turner et al. (2008). Furthermore, the X-ray absorption line depths are sensitive to changes in the covering fraction; from close examination of RGS high resolution spectra, there is no evidence to 
suggest that the covering fraction of phase B changed between observations.

The only parameters that indicate a change during the low flux XMM-Newton Obs. 3 are the ionisation parameter $\xi$ of phase A and the column density $N_{\mathrm{H}}$ of phase B. We find $\log \xi \sim$ 0.9 and $N_{\mathrm{H}} \sim 3 \times 10^{22} \mathrm{~cm}^{-2}$ in Obs. 3, whereas in the other three observations they are $\sim 1.0$ and $\sim 2 \times 10^{22} \mathrm{~cm}^{-2}$, respectively. However, these changes in the warm absorber parameters are very small compared to those in the continuum parameters: power-law slope $\Gamma$ from $\sim 1.8$ to 1.7 in Obs. 3, and normalisation from $\sim 3$ to $2 \times 10^{51}$ photons s $\mathrm{seV}^{-1}$ at $1 \mathrm{keV}$; modified black body temperature from $\sim 190$ to $210 \mathrm{eV}$ in Obs. 3, and normalisation (emitting area times square root of electron density) from $\sim 1.5$ to $0.5 \times 10^{33} \mathrm{~cm}^{0.5}$.

The power-law contribution to the continuum heavily outweighs the modified black body in all our four observations and is responsible for nearly all of the continuum variability. From the X-ray lightcurve (Fig. 1) and spectrum (Fig. 2), the variation in Obs. 3 is larger in the soft X-ray $(0.2-2.0 \mathrm{keV})$ energy band than in the hard X-ray $(2.0-10.0 \mathrm{keV})$ band. This type of variability has been seen in other X-ray observations of Seyfert 1 AGN (such as NGC 7469, Blustin et al. 2003), in which the source is softer when brighter.

\section{Conclusions}

1. We have studied the warm absorber in NGC 3516 by analysing in detail EPIC-pn and RGS high resolution spectra from four observations made in October 2006 by XMM-Newton. The warm absorber consists of three phases of ionisation: phase A ( $\log \xi \sim 0.9)$, phase B ( $\log \xi \sim 2.4)$ and phase $\mathrm{C}(\log \xi \sim 3.0)$ in increasing order of ionisation. Phase A has a hydrogen column density of $\sim 0.4 \times 10^{22} \mathrm{~cm}^{-2}$, which is smaller than those of the other two phases $\left(\sim 1-2 \times 10^{22} \mathrm{~cm}^{-2}\right)$. There is evidence that the lowerionisation phase $\mathrm{A}$ is outflowing at $\sim 100 \mathrm{~km} \mathrm{~s}^{-1}$, whereas the two higher-ionisation phases are outflowing faster at around 1000 to $1500 \mathrm{~km} \mathrm{~s}^{-1}$.

2. Phase B covers about $60 \%$ of the source continuum. We investigated whether variation in the covering fraction of phase B could account for the observed flux and spectral variability in Obs. 3 (as claimed by Turner et al. 2008). We found that (1) the covering fraction does not show significant variation between observations; (2) even if the covering fraction is altered significantly, this does not properly account for the observed variability in the EPIC-pn and especially RGS spectra. This makes a clumpy disk-wind scenario a rather unfeasible explanation.

3. Similar to Netzer et al. (2002), we conclude that the variably in the 2006 observations presented here (albeit much smaller than in previous observations) is better understood as the consequence of changes in the source continuum emission than in the warm absorber. Our results suggest that the X-ray variability of NGC 3516 (and by inference, possibly that of other AGN where a similar behaviour has been observed) cannot be reduced to occultation and absorption effects, such as proposed by Turner et al. (2008) (see also review by Turner \& Miller 2009, and references therein); rather, the variability is likely to arise in a scenario where intrinsic changes of the continuum and ionisation state are also important. In this context, a careful analysis of the soft
$\mathrm{X}$-ray spectrum at high resolution, in combination with data in the Fe Ka regime, can provide essential constraints and clues to the source's physical behaviour.

Acknowledgements. This work is based on observations obtained with $X M M-N e w t o n$, an ESA science mission with instruments and contributions directly funded by ESA member states and the USA (NASA). Missagh Mehdipour acknowledges the support of a PhD studentship awarded by the UK Science and Technology Facilities Council (STFC). We thank the referee, Elisa Costantini, for all her useful comments that improved the paper.

\section{References}

Bautista, M. A., \& Kallman, T. R. 2001, ApJS, 134, 139 Behar, E., Sako, M., \& Kahn, S. M. 2001, ApJ, 563, 497

Blustin, A. J., Branduardi-Raymont, G., Behar, E., et al. 2002, A\&A, 392, 453

Blustin, A. J., Branduardi-Raymont, G., Behar, E., et al. 2003, A\&A, 403, 481

Blustin, A. J., Page, M. J., Fuerst, S. V., Branduardi-Raymont, G., \& Ashton, C. E. 2005, A\&A, 431, 111

Branduardi-Raymont, G., Sako, M., Kahn, S. M., et al. 2001, A\&A, 365, L140 Chakravorty, S., Kembhavi, A. K., Elvis, M., \& Ferland, G. 2009, MNRAS, 393, 83

Costantini, E., Nicastro, F., Fruscione, A., et al. 2000, ApJ, 544, 283

Costantini, E., Kaastra, J. S., Arav, N., et al. 2007, A\&A, 461, 121

George, I. M., Turner, T. J., Mushotzky, R., Nandra, K., \& Netzer, H. 1998, ApJ, 503, 174

Halpern, J. P. 1984, ApJ, 281, 90

Kaastra, J. S., \& Barr, P. 1989, A\&A, 226, 59

Kaastra, J. S., Mewe, R., \& Nieuwenhuijzen, H. 1996, in UV and X-ray Spectroscopy of Astrophysical and Laboratory Plasmas, ed. K. Yamashita, \& T. Watanabe, 411

Kaastra, J. S., Mewe, R., Liedahl, D. A., Komossa, S., \& Brinkman, A. C. 2000, A\&A, 354, L83

Kaastra, J. S., Steenbrugge, K. C., Raassen, A. J. J., et al. 2002, A\&A, 386, 427 Kaastra, J. S., Lieu, R., Tamura, T., Paerels, F. B. S., \& den Herder, J. W. 2003, A\&A, 397, 445

Kaastra, J. S. 2008, Astron. Nachr., 329, 162

Kaastra, J. S., Mewe, R., \& Raassen, A. J. J. 2009, SPEX User's Manual, http://www . sron.nl/files/HEA/SPEX/manuals/manual . pdf

Kalberla, P. M. W., Burton, W. B., Hartmann, D., et al. 2005, A\&A, 440, 775

Keel, W. C. 1996, AJ, 111, 696

Kriss, G. A., Krolik, J. H., Otani, C., et al. 1996, ApJ, 467, 629

Krolik, J. H., McKee, C. F., \& Tarter, C. B. 1981, ApJ, 249, 422

Laor, A. 1991, ApJ, 376, 90

Lodders, K. 2003, ApJ, 591, 1220

Markowitz, A., Edelson, R., \& Vaughan, S. 2003, ApJ, 598, 935

Markowitz, A., Reeves, J. N., Miniutti, G., et al. 2008, PASJ, 60, 277

Miniutti, G., \& Fabian, A. C. 2004, MNRAS, 349, 1435

Nandra, K., \& Pounds, K. A. 1992, Nature, 359, 215

Nandra, K., Fabian, A. C., George, I. M., et al. 1993, MNRAS, 260, 504

Netzer, H., Chelouche, D., George, I. M., et al. 2002, ApJ, 571, 256

Nikołajuk, M., Czerny, B., Ziółkowski, J., \& Gierliński, M. 2006, MNRAS, 370, 1534

Reeves, J. N., O’Brien, P. T., \& Ward, M. J. 2003, ApJ, 593, L65

Reynolds, C. S. 1997, MNRAS, 286, 513

Sako, M., Kahn, S. M., Behar, E., et al. 2001, A\&A, 365, L168

Sako, M., Kahn, S. M., Branduardi-Raymont, G., et al. 2003, ApJ, 596, 114

Smith, R. A. N. 2009, Ph.D. Thesis, Mullard Space Science Laboratory,

University College London, Holmbury St. Mary, Dorking, Surrey RH5 6NT, UK

Smith, R. A. N., Page, M. J., \& Branduardi-Raymont, G. 2008, A\&A, 490, 103

Steenbrugge, K. C., Kaastra, J. S., de Vries, C. P., \& Edelson, R. 2003, A\&A, 402, 477

Steenbrugge, K. C., Kaastra, J. S., Crenshaw, D. M., et al. 2005, A\&A, 434, 569

Turner, T. J., \& Miller, L. 2009, A\&A Rev., 17, 47

Turner, T. J., Nandra, K., George, I. M., Fabian, A. C., \& Pounds, K. A. 1993, ApJ, 419, 127

Turner, T. J., Mushotzky, R. F., Yaqoob, T., et al. 2002, ApJ, 574, L123

Turner, T. J., Kraemer, S. B., George, I. M., Reeves, J. N., \& Bottorff, M. C. 2005, ApJ, 618, 155

Turner, T. J., Reeves, J. N., Kraemer, S. B., \& Miller, L. 2008, A\&A, 483, 161

Wu, X.-B., \& Han, J. L. 2001, ApJ, 561, L59

Zhang, T.-Z., \& Wu, X.-B. 2002, Chinese J. Astron. Astrophys., 2, 487 